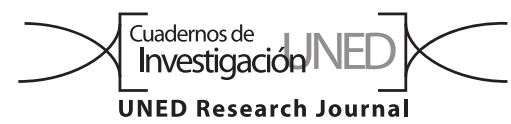

\title{
¿Se puede "secuestrar" carbono a la vez que se conserva la biodiversidad de los bosques? Revisión de la evidencia científica y recomendaciones
}

\author{
Andrea G. Vincent \\ 1. Escuela de Biología, Universidad de Costa Rica, 2060 San José, Costa Rica; andrea.vincent@ucr.ac.cr
}

Recibido 07-IX-2018 • Corregido 11-XII-2018 • Aceptado 14-I-2019

\begin{abstract}
Can carbon be "sequestered" while preserving the biodiversity of forests? Review of scientific evidence and recommendations. Tropical forests store a great portion of the terrestrial carbon in their biomass and initiatives such as REDD+ (Reducing Emissions from Deforestation and forest Degradation) aim to avoid the emission of this carbon by protecting forests. Nevertheless, not all carbon-rich forests are also rich in biodiversity. Here, I summarize existing information on the carbon-biodiversity relationship in tropical forests, highlighting the need for REDD+ to explicitly consider biodiversity conservation, not just carbon sequestration.
\end{abstract}

Key words: REDD+, carbon-biodiversity relationship, tropical forest, soil, mitigation, climate change.
RESUMEN: Los bosques tropicales almacenan gran parte del carbono terrestre en su biomasa e iniciativas como REDD+ (Reducción de Emisiones por Deforestación y Degradación del Bosque y más) buscan evitar la liberación de este carbono por medio de la protección de los bosques. Sin embargo, no todos los bosques ricos en carbono son ricos en biodiversidad. Aquí, sintetizo la información existente sobre la relación entre la cantidad de carbono almacenado y la biodiversidad de árboles en bosques tropicales, enfatizando la importancia de que REDD+ explícitamente maximice la conservación de la biodiversidad, no solo del carbono.

Palabras clave: REDD+, relación biodiversidad-carbono, bosque tropical, suelo, mitigación, cambio climático.
Los bosques tropicales contienen $25 \%$ del carbono terrestre del planeta en su biomasa (Bonan, 2008) y producen $34 \%$ de la productividad primaria gruesa (Beer et al., 2010). Por ende, hay un fuerte interés global en evitar su deforestación y degradación con el fin de evitar la liberación de su carbono a la atmósfera (Miles \& Kapos, 2008). Uno de los principales mecanismos financieros de mitigación de cambio climático en los trópicos es el programa REDD+ (Reducción de Emisiones por Deforestación y Degradación del bosque y más) de la Convención Marco de Naciones Unidas sobre el Cambio Climático (CMNUCC), y diversas entidades se han comprometido a donar $\$ 10$ mil millones de dólares para esta iniciativa desde el 2004 (Nakhooda et al., 2014), sobrepasando el financiamiento para conservación "tradicional", i.e. enfocada en conservar la biodiversidad (James, Gaston \& Balmford, 2001). La iniciativa REDD+ reconoce la importancia de proteger la biodiversidad al mismo tiempo que el carbono de los bosques, para evitar consecuencias negativas para la naturaleza (Phelps, Webb \& Adams, 2012). Sin embargo, esto requeriría que la biodiversidad estuviera positivamente relacionada con la densidad de carbono, lo cual no siempre es el caso. En esta nota examino la evidencia científica sobre la naturaleza de la relación carbono-biodiversidad de árboles en bosques tropicales, con el fin de apoyar a los y las tomadoras de decisiones en la selección de iniciativas que maximicen la protección del carbono y de la biodiversidad.

El crecimiento vegetal en los bosques tropicales tiende a estar correlacionado con su biomasa (Chisholm et al., 2013), la cual está relacionada con carbono, ya que aproximadamente el $50 \%$ de la biomasa vegetal es carbono. Las hipótesis sobre la relación biodiversidad-crecimiento son útiles para examinar los mecanismos responsables de una posible relación positiva entre biodiversidad y carbono. Por ejemplo, la hipótesis de la complementariedad de nichos (Tilman et al., 2001) postula que una alta biodiversidad aumenta la eficiencia de uso de recursos distintos de un lugar, ya que hay más diversidad de formas de explotarlos, lo cual aumenta el crecimiento total de la comunidad. Otras hipótesis proponen que cuando hay mayor diversidad hay más probabilidad de encontrar 
ciertas especies que naturalmente facilitan el crecimiento de otras, i.e. hipótesis de la facilitación (Hooper et al., 2005), más probabilidad de muestrear una especie altamente productiva que aumente el promedio de productividad de la comunidad entera, i.e. hipótesis de selección (Loreau \& Hector, 2001; Turnbull et al., 2013), y más probabilidad de que si la abundancia de una especie disminuye por alguna perturbación, otra pueda reemplazarla en sus funciones y seguir creciendo, i.e. hipótesis del seguro (Yachi \& Loreau, 1999; Isbell et al., 2011). También se cree que la diversidad reduce los efectos de patógenos especialistas debido a una "dilución" del impacto negativo de los patógenos en una comunidad vegetal diversa, lo cual aumenta la productividad primaria (Schnitzer et al., 2011). Cabe destacar que estas hipótesis no son mutualmente exclusivas.

Muchos estudios apoyan una relación positiva entre biodiversidad y productividad, mediada a través de la complementariedad de nichos y otros de los mecanismos mencionados arriba (Balvanera et al., 2006; Cardinale et al., 2012, 2011; Paquette \& Messier, 2011; Tilman, Isbell, \& Cowles, 2014). Sin embargo, la gran mayoría de estos estudios se han realizado en comunidades herbáceas (Cardinale et al., 2012, 2011; Tilman et al., 2014) y bosques poco diversos (Balvanera et al., 2006; Butterfield \& Suding, 2013; Paquette \& Messier, 2011) en zonas templadas. En los últimos años se han publicado varios estudios que analizan la relación biodiversidad-crecimiento para árboles de bosques tropicales, utilizando parcelas forestales permanentes. De los estudios que consideran parcelas con bosques maduros protegidos y a escalas espaciales pequeñas $(\sim 0.04 \mathrm{ha}$, o $20 \times$ $20 \mathrm{~m})$ y grandes $(\sim 1 \mathrm{ha}, 100 \times 100 \mathrm{~m})$ en el mismo estudio, todos encuentran relaciones positivas entre diversidad y crecimiento (Chisholm et al., 2013; Poorter et al., 2015; Sullivan et al., 2017).

Sin embargo, a escalas espaciales mayores ( $>1 \mathrm{ha}$, $100 \times 100 \mathrm{~m})$ en bosques no perturbados y con parcelas distribuidas a nivel continental o pantropical, algunos muestran relaciones positivas entre biodiversidad y carbono (Cavanaugh et al., 2014; Poorter et al., 2015), otros no (Sullivan et al., 2017), o bien reportan resultados mixtos (Chisholm et al., 2013), lo cual ha generado un aumento de actividad en esta área de investigación (van der Sande et al., 2017a). Sin embargo, parece ser que el nivel de heterogeneidad ambiental entre parcelas es determinante, porque pueden confundirse los impactos del ambiente con el efecto de la biodiversidad sobre el carbono (van der Sande et al., 2017a). Por ejemplo, algunos de los estudios muestran grandes variaciones en precipitación anual y/o mucha variación estacional (Poorter et al., 2015; van der Sande et al., 2017b), otros muestran diferencias amplias en temperatura por incluir sitios templados y tropicales (Chisholm et al., 2013) o considerables gradientes de temperatura debido a una alta variabilidad en elevación (Cavanaugh et al., 2014).

Finalmente, el nivel de perturbación en las parcelas estudiadas juega un papel importante, ya que en ciertos casos, una relación positiva diversidad-carbono que tiene lugar a pequeña escala no se observa cuando el bosque ha sido deforestado (van der Sande et al., 2018), o se da pero solo en los estadios sucesionales tempranos, y desaparece cuando los bosques alcanzan una biomasa específica (Ferreira et al., 2018). Lo que queda claro es que no hay una relación universalmente positiva entre carbono y biodiversidad de árboles en los bosques tropicales (Sullivan et al., 2017). Considerar esa realidad es fundamental a la hora de implementar iniciativas REDD+, con el fin de no ocasionar pérdidas de biodiversidad aplicando un enfoque centrado en carbono (Anderson-Teixeira, 2018).

¿Qué implicaciones tienen estos hallazgos? ¿Acaso quieren decir que debemos escoger entre secuestrar carbono y proteger la biodiversidad? Aunque no hay evidencia de una relación universalmente positiva entre biodiversidad y carbono para árboles tropicales, sí hay ciertos bosques tropicales que tienen alta densidad de carbono y alta biodiversidad al mismo tiempo, principalmente en el sudeste asiático (Cavanaugh et al., 2014; Sullivan et al., 2017) así como en bosques de estadios sucesionales tempranos. Los proyectos enfocados en la restauración de reservas de carbono en bosques secundarios muy perturbados podrían generar beneficios simultáneos carbono-biodiversidad, aunque tanto el carbono total como la biodiversidad total serían menores que en bosques intactos. Por otro lado, proyectos REDD+ enfocados en bosques ricos en carbono en la mayoría de los casos no tienen la garantía de conservar los bosques más diversos (Di Marco, Watson, Currie, Possingham, \& Venter, 2018; Ferreira et al., 2018; Sullivan et al., 2017). Una opción para evitar esta situación sería que los proyectos aceptaran conservar un poco menos del carbono máximo posible para conservar significativamente más biodiversidad (Anderson-Teixeira, 2018). En algunos casos, se ha calculado que renunciar a un $1 \%$ de las ganancias que se podrían recaudar por el carbono secuestrado en un lugar podría ayudar a conservar ocho veces más especies grandes de árboles, importante considerando que $1 \%$ es una cifra menor al valor de incertidumbre en las mediciones de carbono (Anderson-Teixeira, 2018; Ferreira et al., 2018). De esta manera la conservación de bosques en ciertos casos podría aumentar la protección de la biodiversidad sin incurrir en un costo significativo de carbono. 
A la hora de conservar bosques, es necesario incluir variables relevantes en términos de conservación, y no solamente el contenido de carbono de un sitio, que es lo que ultimadamente incentiva REDD+ (AndersonTeixeira, 2018). La diversidad de taxones como vertebrados también puede estar positivamente (Ferreira et al., 2018; Sobral et al., 2017) o negativamente (Beaudrot et al., 2016; Ferreira et al., 2018) relacionada con el carbono, dependiendo de la escala espacial considerada y muchos otros factores. La diversidad funcional es importante también, ya que hay comunidades taxonómicamente poco diversas pero que realizan funciones vitales para los ecosistemas, e.g. comunidades de páramo o desiertos fríos, que albergan especies únicas y brindan servicios ecosistémicos clave. Además, no todas las reservas de carbono son epígeas, hay mucho carbono en suelos en ecosistemas tropicales como turberas (Draper et al., 2014), que actualmente no está considerado en los análisis mencionados anteriormente. Finalmente, es de crucial importancia considerar también variables sociales, económicas, políticas y de género, ya que si no se hace REDD+ podría tener efectos negativos sobre las comunidades humanas que viven dentro o cerca de los bosques dentro de esquemas REDD+ (Larson et al., 2013; Moeliono et al., 2014; Chomba et al., 2015; Larson et al., 2018; Myers et al., 2018). Utilizar esta gama más amplia de variables es un gran reto en países centroamericanos, ya que muchas veces no hay presupuesto para realizar inventarios detallados de carbono y todas esas variables de biodiversidad, para cada proyecto. Generar esta información aumentaría el costo de REDD+ (Gardner et al., 2012; Phelps et al., 2012) pero es necesaria para poder implementar proyectos de protección de carbono en bosques y al mismo tiempo conservar la biodiversidad asociada a ellos. Igual o más importante que estos aspectos técnicos, sin embargo, es asegurarse que REDD+ no reproduzca las mismas estructuras de acceso a la comercialización de los recursos forestales que actualmente causan la deforestación.

\section{REFERENCIAS}

Anderson-Teixeira, K. J. (2018). Prioritizing biodiversity and carbon. Nature Climate Change 8, 667-668.

Balvanera, P., Pfisterer, A. B., Buchmann, N., He, J., Nakashizuka, T., Raffaelli, D., \& Schmid, B. (2006). Quantifying the evidence for biodiversity effects on ecosystem functioning and services. Ecology Letters, 9(10), 1146-1156.

Beaudrot, L., Kroetz, K., Alvarez-Loayza, P., Amaral, I., Breuer, T., Fletcher, C., ... Marshall, A. R. (2016). Limited carbon and biodiversity co-benefits for tropical forest mammals and birds. Ecological Applications, 26(4), 1098-1111.
Beer, C., Reichstein, M., Tomelleri, E., Ciais, P., Jung, M., Carvalhais, N., ... Bonan, G. B. (2010). Terrestrial gross carbon dioxide uptake: global distribution and covariation with climate. Science, 329, 834-838.

Bonan, G. B. (2008). Forests and climate change: forcings, feedbacks, and the climate benefits of forests. Science, 320(5882), 1444-1449.

Butterfield, B. J., \& Suding, K. N. (2013). Single-trait functional indices outperform multi-trait indices in linking environmental gradients and ecosystem services in a complex landscape. Journal of Ecology, 101(1), 9-17.

Cardinale, B. J., Duffy, J. E., Gonzalez, A., Hooper, D. U., Perrings, C., Venail, P., ... Wardle, D. A. (2012). Biodiversity loss and its impact on humanity. Nature, 486(7401), 59.

Cardinale, B. J., Matulich, K. L., Hooper, D. U., Byrnes, J. E., Duffy, E., Gamfeldt, L., ... Gonzalez, A. (2011). The functional role of producer diversity in ecosystems. American Journal of Botany, 98(3), 572-592.

Cavanaugh, K. C., Gosnell, J. S., Davis, S. L., Ahumada, J., Boundja, P., Clark, D. B., ... Rovero, F. (2014). Carbon storage in tropical forests correlates with taxonomic diversity and functional dominance on a global scale. Global Ecology and Biogeography, 23(5), 563-573.

Chisholm, R. A., Muller-Landau, H. C., Rahman, K. A., Bebber, D. P., Bin, Y., Bohlman, S. A., ... Butt, N. (2013). Scaledependent relationships between tree species richness and ecosystem function in forests. Journal of Ecology, 101(5), 1214-1224.

Chomba, S., Kariuki, J., Lund, J. F., \& Sinclair, F. (2016). Roots of inequity: How the implementation of REDD+ reinforces past injustices. Land Use Policy, 50, 202-213.

Di Marco, M., Watson, J. E. M., Currie, D. J., Possingham, H. P., \& Venter, O. (2018). The extent and predictability of the biodiversity-carbon correlation. Ecology Letters, 21(3), 365-375.

Draper, F. C., Roucoux, K. H., Lawson, I. T., Mitchard, E. T. A., Coronado, E. N. H., Lähteenoja, O., ... Baker, T. R. (2014). The distribution and amount of carbon in the largest peatland complex in Amazonia. Environmental Research Letters, 9(12), 124017.

Ferreira, J., Lennox, G. D., Gardner, T. A., Thomson, J. R., Berenguer, E., Lees, A. C., ... Louzada, J. (2018). Carbonfocused conservation may fail to protect the most biodiverse tropical forests. Nature Climate Change, 8(8), 744.

Gardner, T. A., Burgess, N. D., Aguilar-Amuchastegui, N., Barlow, J., Berenguer, E., Clements, T., ... Kapos, V. (2012). A framework for integrating biodiversity concerns into national REDD+ programmes. Biological Conservation, 154, 61-71.

Hooper, D. U., Chapin, F. S., Ewel, J. J., Hector, A., Inchausti, P., Lavorel, S., ... Naeem, S. (2005). Effects of biodiversity on ecosystem functioning: a consensus of current knowledge. Ecological Monographs, 75(1), 3-35. 
Isbell, F., Calcagno, V., Hector, A., Connolly, J., Harpole, W. S., Reich, P. B., ... Van Ruijven, J. (2011). High plant diversity is needed to maintain ecosystem services. Nature, 477(7363), 199.

James, A., Gaston, K. J., \& Balmford, A. (2001). Can we afford to conserve biodiversity? AIBS Bulletin, 51(1), 43-52.

Larson, A. M., Brockhaus, M., Sunderlin, W. D., Duchelle, A., Babon, A., Dokken, T., ... \& Huynh, T. B. (2013). Land tenure and REDD+: The good, the bad and the ugly. Global Environmental Change, 23(3), 678-689.

Larson, A. M., Solis, D., Duchelle, A. E., Atmadja, S., Resosudarmo, I. A. P., Dokken, T., \& Komalasari, M. (2018). Gender lessons for climate initiatives: A comparative study of REDD+ impacts on subjective wellbeing. World Development, 108, 86-102

Loreau, M., \& Hector, A. (2001). Partitioning selection and complementarity in biodiversity experiments. Nature, 412(6842), 72.

Moeliono, M., Gallemore, C., Santoso, L., Brockhaus, M., \& Di Gregorio, M. (2014). Information networks and power: confronting the "wicked problem" of REDD+ in Indonesia. Ecology and Society, 19(2), 9.

Miles, L., \& Kapos, V. (2008). Reducing greenhouse gas emissions from deforestation and forest degradation: global land-use implications. Science, 320(5882), 1454-1455.

Myers, R., Larson, A. M., Ravikumar, A., Kowler, L. F., Yang, A., \& Trench, T. (2018). Messiness of forest governance: How technical approaches suppress politics in REDD+ and conservation projects. Global Environmental Change, 50, 314-324.

Nakhooda, S., Norman, M., Barnard, S., Watson, C., Greenhill, R., Caravani, A., ... Banton, G. (2014). Climate finance: Is it making a difference. A Review of the Effectiveness of Multilateral Climate Funds. London: Overseas Development Institute.

Paquette, A., \& Messier, C. (2011). The effect of biodiversity on tree productivity: from temperate to boreal forests. Global Ecology and Biogeography, 20(1), 170-180.

Phelps, J., Webb, E. L., \& Adams, W. M. (2012). Biodiversity co-benefits of policies to reduce forest-carbon emissions. Nature Climate Change, 2(7), 497.
Poorter, L., Van Der Sande, M. T., Thompson, J., Arets, E., Alarcón, A., Álvarez-Sánchez, J., ... Boit, A. (2015). Diversity enhances carbon storage in tropical forests. Global Ecology and Biogeography, 24(11), 1314-1328.

Schnitzer, S. A., Klironomos, J. N., HilleRisLambers, J., Kinkel, L. L., Reich, P. B., Xiao, K., ... Mangan, S. A. (2011). Soil microbes drive the classic plant diversity-productivity pattern. Ecology, 92(2), 296-303.

Sobral, M., Silvius, K. M., Overman, H., Oliveira, L. F. B., Raab, T. K., \& Fragoso, J. M. V. (2017). Mammal diversity influences the carbon cycle through trophic interactions in the Amazon. Nature Ecology \& Evolution, 1(11), 1670.

Sullivan, M. J. P., Talbot, J., Lewis, S. L., Phillips, O. L., Qie, L., Begne, S. K., ... Lopez-Gonzalez, G. (2017). Diversity and carbon storage across the tropical forest biome. Scientific Reports, 7, 39102.

Tilman, D., Isbell, F., \& Cowles, J. M. (2014). Biodiversity and ecosystem functioning. Annual Review of Ecology, Evolution, and Systematics, 45, 471-493.

Tilman, D., Reich, P. B., Knops, J., Wedin, D., Mielke, T., \& Lehman, C. (2001). Diversity and productivity in a long-term grassland experiment. Science, 294(5543), 843-845.

Turnbull, L. A., Levine, J. M., Loreau, M., \& Hector, A. (2013). Coexistence, niches and biodiversity effects on ecosystem functioning. Ecology Letters, 16, 116-127.

van der Sande, M.T., Arets, E. J. M. M., Peña-Claros, M., Hoosbeek, M. R., Cáceres-Siani, Y., van der Hout, P., \& Poorter, L. (2018). Soil fertility and species traits, but not diversity, drive productivity and biomass stocks in a Guyanese tropical rainforest. Functional Ecology, 32(2), 461-474.

van der Sande, M. T., Poorter, L., Kooistra, L., Balvanera, P., Thonicke, K., Thompson, J., ... Mora, F. (2017a). Biodiversity in species, traits, and structure determines carbon stocks and uptake in tropical forests. Biotropica, 49(5), 593-603.

van der Sande, M. T., Peña-Claros, M., Ascarrunz, N., Arets, E. J. M. M., Licona, J. C., Toledo, M., \& Poorter, L. (2017b). Abiotic and biotic drivers of biomass change in a Neotropical forest. Journal of Ecology, 105(5), 1223-1234.

Yachi, S., \& Loreau, M. (1999). Biodiversity and ecosystem productivity in a fluctuating environment: the insurance hypothesis. Proceedings of the National Academy of Sciences, 96(4), 1463-1468. 